\title{
ANÁLISE QUANTITATIVA DA SATISFAÇÃO PROFISSIONAL DOS ENFERMEIROS QUE ATUAM NO PERÍODO NOTURNO
}

\author{
Rosângela Marion da Silvaํ, Carmem Lúcia Colomé Beck², Laura de Azevedo Guido³, Luis Felipe Dias Lopes4, \\ José Luís Guedes dos Santos ${ }^{5}$
}

${ }^{1}$ Mestre em Enfermagem. Enfermeira do Hospital Universitário de Santa Maria. Rio Grande do Sul, Brasil. E-mail: cucasma@ terra.com.br

${ }^{2}$ Doutora em Enfermagem. Professor Associado do Departamento de Enfermagem Universidade Federal de Santa Maria (UFSM). Rio Grande do Sul, Brasil. E-mail: carmembeck@smail.ufsm.br

${ }^{3}$ Doutora em Enfermagem. Professor Adjunto do Departamento de Enfermagem da UFSM. Rio Grande do Sul, Brasil. E-mail: lguido@terra.com.br

${ }^{4}$ Doutor em Engenharia de Produção. Professor Adjunto do Departamento de Estatística da UFSM. Rio Grande do Sul, Brasil. E-mail: lflopes@smail.ufsm.br

${ }^{5}$ Mestrando em Enfermagem pelo Programa de Pós-Graduação em Enfermagem da Universidade Federal do Rio Grande do Sul. Bolsista Capes. Rio Grande do Sul, Brasil. E-mail: joseenfermagem@yahoo.com.br

RESUMO: Estudo de campo, exploratório, descritivo, com abordagem quantitativa que objetivou caracterizar os enfermeiros que trabalham no período noturno de um Hospital Universitário do Estado do Rio Grande do Sul, bem como identificar o nível real de satisfação profissional em relação a seis componentes do trabalho: autonomia, interação, status profissional, requisitos do trabalho, normas organizacionais e remuneração. Na coleta dos dados foram utilizados um questionário para caracterização sociodemográfica e outro para o Índice de Satisfação Profissional. Os dados foram coletados no período de março a abril de 2008 e processados no programa estatístico Statistical Analisys System versão 9.1. Participaram 42 enfermeiros, majoritariamente do sexo feminino (90,48\%), faixa etária maior que 41 anos de idade (64,28\%), estado civil casado ou com companheiro $(64,29 \%)$, com filhos (80,96\%) e que escolheram trabalhar nesse turno $(90,48 \%)$. A autonomia foi considerada o componente de maior nível real de satisfação profissional enquanto normas organizacionais o de menor nível.

DESCRITORES: Satisfação no emprego. Trabalho noturno. Enfermagem.

\section{QUANTITATIVE ANALYSIS OF NIGHT-SHIFT NURSES' PROFESSIONAL SATISFACTION}

\begin{abstract}
This exploratory, quantitative, and descriptive study aimed to characterize the night-shift nurses in a University Hospital in Rio Grande do Sul, Brazil, as well as to identify the actual professional satisfaction rate regarding six work components: autonomy, interaction, professional status, task requirements, organizational policies, and pay. For data collection, one survey was used in order for socio-demographic characterization and another to characterize the Work Satisfaction Index. The data was collected in March and April of 2008 and processed in the Statistical Analysis System software, version 9.1. The sample was composed of 42 participating nurses, mostly female (90.48\%), aged more than 41 years (64.28\%), either married or living with a partner (64.29\%), with children (80.96\%) and who chose to work in this shift $(90.48 \%)$. Autonomy was considered the component which reached the highest level of real satisfaction, while organizational policies received the lowest level of satisfaction.
\end{abstract}

DESCRIPTORS: Job satisfaction. Night work. Nursing.

\section{ANÁLISIS CUANTITATIVO DE LA SATISFACCIÓN PROFESIONAL DE LOS ENFERMEROS QUE ACTÚAN EN EL PERÍODO NOCTURNO}

RESUMEN: Estudio de campo, exploratorio descriptivo, de carácter cuantitativo, cuyo objetivo fue caracterizar los enfermeros que ejercen actividades laborales en el período nocturno en un Hospital Universitario de la provincia de Rio Grande do Sul, e identificar el nivel real de satisfacción profesional relacionado a seis componentes del trabajo: autonomía, interacción, estatus profesional, requisitos del trabajo, normas organizacionales y remuneración. Para la recolección de los datos fueron utilizados dos cuestionarios: uno para la caracterización sociodemográfica y otro para el Índice de Satisfacción Profesional. Los datos, recolectados en marzo y abril de 2008, fueron procesados en el programa estadístico Statistical Analisys System versión 9.1. Participaron 42 enfermeros, la mayoría del sexo femenino $(90,48 \%)$, ubicados en la franja etaria superior a 41 años $(64,28 \%)$, estado civil casado o con compañero $(64,29 \%)$, con hijos $(80,96 \%)$ y que eligieron trabajar en este turno $(90,48 \%)$. La autonomía fue el componente que alcanzó el mayor nivel real de satisfacción profesional, mientras que las normas organizacionales, el menor nivel.

DESCRIPTORES: Satisfacción en el trabajo. Trabajo nocturno. Enfermería. 


\section{INTRODUÇÃO}

Nas últimas décadas, as transformações econômicas e sociais decorrentes do processo de globalização têm alterado, consideravelmente, a relação entre o homem e o seu trabalho. Busca-se, cada vez mais, grande produtividade, associada a um baixo custo de produção, no intuito de obter produtos altamente competitivos no cenário capitalista. Diante desse cenário, observa-se o aumento dos ritmos e cargas de trabalho em detrimento da satisfação dos trabalhadores em executar as tarefas, o que pode repercutir na sua qualidade vida, interferindo no processo saúde/doença.

Em se tratando do trabalho de enfermagem desempenhado, especialmente, no cenário hospitalar, há referência de que este é diferenciado dos demais trabalhos porque é contínuo, desgastante, exaustivo e desenvolvido a partir de uma relação interpessoal muito próxima com o paciente sob seus cuidados. Também é capaz de proporcionar sentimentos como alegria, satisfação e prazer aos trabalhadores, sem os quais seria praticamente impossível exercer a profissão. ${ }^{1}$

O processo de trabalho do enfermeiro no período noturno possibilita, em muitas situações, tomar decisões, atuar com autonomia, o que pode ser um fator que contribui para a satisfação no trabalho. Por outro viés, a dificuldade em participar de espaços promovidos pela instituição no período diurno como reuniões, palestras, atividades de educação permanente ou até mesmo para conhecimento ou apresentação de novas normas, rotinas ou equipamentos, pode ser considerada desgastante e angustiante para esse trabalhador no que tange às normas organizacionais, o que pode não favorecer a satisfação com relação a esse componente do trabalho.

A satisfação no trabalho* resulta da complexa e dinâmica interação das condições gerais de vida, das relações de trabalho, do processo de trabalho e do controle que os trabalhadores possuem sobre suas condições de vida e de trabalho. ${ }^{2}$

Nesse sentido, a satisfação é um processo dinâmico que pode ter influência tanto da organização do trabalho quanto da vida social, ou seja, o trabalhador não chega ao seu local de trabalho como uma máquina nova; ele possui uma história, o que o torna um indivíduo com características únicas e pessoais. ${ }^{3}$

Nessa lógica, a realização das atividades no noturno, período em que o organismo opta pelo descanso, associado ao esforço em manter-se acordado e às expectativas pessoais do trabalhador, são fatores que podem deflagrar sentimentos de maior ou menor satisfação no trabalho com relação a determinados aspectos do processo de trabalho, sendo, portanto, processo ativo e complexo.

Essas considerações preliminares, associada à leitura e a vivência profissional no trabalho noturno, incitaram ao questionamento sobre a relação do trabalhador com a atividade noturna: qual dos componentes do trabalho, entendidos como autonomia, interação, status profissional, requisitos do trabalho, normas organizacionais $\mathrm{e}$ remuneração, proporciona maior e menor nível real de satisfação profissional para o enfermeiro que atua no noturno?

A partir deste questionamento, esse artigo, recorte de uma dissertação de Mestrado, defendida no Programa de Pós Graduação em Enfermagem da Universidade Federal de Santa Maria (UFSM-RS), ${ }^{4}$ tem como objetivo caracterizar os enfermeiros que trabalham no período noturno de um Hospital Universitário e identificar o nível real de satisfação profissional.

\section{CASUÍSTICA E MÉTODO}

Esse artigo trata de uma pesquisa exploratória, descritiva, com abordagem quantitativa que tem como estratégia de pesquisa verificar os resultados por meio de objetivos previamente definidos e está sendo cada vez mais utilizada, tendo em vista que a maior parte dos estudos avaliativos preocupa-se em mensurar o impacto ou efeito da intervenção. ${ }^{5}$

O cenário da pesquisa foi um Hospital Universitário do Estado do Rio Grande do Sul. Essa instituição é referência regional em alta complexidade e está localizada em uma cidade de, aproximadamente, 270 mil habitantes. Os atendimentos prestados à comunidade são realizados nos 309 leitos, distribuídos pelas unidades de internação, unidades de tratamento intensivo, ambulatórios, salas para atendimento de emergência e pronto atendimento.

A partir dos critérios de inclusão na pesquisa, ou seja, atuar há pelo menos um ano no período noturno da instituição pesquisada como enfermeiro e pertencer ao quadro permanente da instituição, somado a exclusão das pessoas que estavam em licença de qualquer natureza por diversos motivos, participaram efetivamente 42 enfermeiros.

* Para este estudo, os termos satisfação no trabalho e satisfação profissional serão utilizados como sinônimos. 
Após a aprovação do projeto pelo Comitê de Ética em Pesquisa ao qual a instituição está vinculada, a pesquisa recebeu parecer favorável sob $\mathrm{N}^{\circ}$ 0012.0.243.000-08. A seguir foi efetuado o contato pessoal com os enfermeiros, informando-os acerca dos objetivos da pesquisa e convidando-os a participar. Após o consentimento, foi entregue o Termo de Consentimento Livre e Esclarecido e solicitada a assinatura em caso de concordância com os termos expostos. Além disso, foram orientados quanto à voluntariedade, a proteção da imagem e garantia do anonimato, quanto ao direito de recusar-se a participar da pesquisa, a retirar o consentimento a qualquer momento sem que disto lhe resultasse em algum prejuízo, conforme os preceitos da Resolução Nº 196/96 do Conselho Nacional de Saúde. ${ }^{6}$

A coleta dos dados foi realizada no período de março a abril de 2008 por um dos pesquisadores. Para tal, foram utilizados dois questionários: um para caracterizar os enfermeiros e outro para avaliar o nível real de satisfação profissional, o Índice de Satisfação Profissional (ISP).

Ressalta-se que foi realizado um pré-teste a fim de verificar possíveis dificuldades no preenchimento dos questionários, sendo convidados, aleatoriamente, três enfermeiros do período noturno que receberam e preencheram os questionários, os quais não demandaram alterações.

O questionário que possibilitou a caracterização dos enfermeiros foi composto por dados como sexo, faixa etária, estado civil, número de filhos, a opção pelo trabalho noturno e o tempo de serviço noturno na instituição.

O ISP foi traduzido, adaptado e validado para a língua portuguesa e para a cultura de enfermeiras brasileiras. ${ }^{7}$ É auto-aplicável e autoexplicativo porque traz na sua estrutura a orientação para o preenchimento e explicação conceitual dos seis componentes:

- autonomia: grau de independência, iniciativa e liberdade, tanto permitido quanto necessário, nas atividades diárias do trabalho;

- interação: oportunidade de contato social e profissional, formal e informal durante o horário de trabalho;

- status profissional: importância ou significância percebida acerca do seu trabalho, tanto no seu ponto de vista como no de outros;

- requisitos do trabalho: tarefas ou atividades que devem ser executadas como parte regular do trabalho;
- normas organizacionais: normas administrativas e procedimentos propostos pelo hospital e administração do serviço de enfermagem;

- remuneração: pagamento em dinheiro e benefícios adicionais recebidos pelo trabalho executado.

Esse questionário é composto por duas partes: a primeira parte (Parte A) consiste em uma lista de 15 pares que combinam os seis componentes do ISP entre si o que leva o respondente a escolher, de cada par, o que mais influencia sobre sua satisfação profissional; a segunda (Parte B), consiste em uma Escala de Atitudes, com 44 afirmações dispostas em uma escala do tipo Likert, de sete pontos, que varia entre discorda inteiramente (7) e concorda inteiramente (1). Ressalta-se que os itens dos componentes: autonomia (8), interação (10), status profissional (7), requisitos do trabalho (6), remuneração (6) e normas organizacionais (7), estão dispostos aleatoriamente na escala, sendo que 22 itens são ordenados positivamente e 22 negativamente.

Os dados, após serem digitados em planilha eletrônica, em forma de banco de dados, foram, posteriormente, processados no programa estatístico Statistical Analisys System (SAS) versão 9.1. O nível de significância estabelecido foi de 5\%. Para avaliar a consistência interna da escala total do ISP foi utilizado o alfa de Cronbach.

Para a análise estatística da Parte A, foi criada uma matriz de freqüência que relaciona quantas vezes cada componente foi escolhido em relação aos outros componentes. As freqüências das respostas foram convertidas em uma matriz de proporção, por meio da divisão do número de vezes que o componente foi escolhido pelo tamanho total do grupo pesquisado. Para a conversão das proporções em desvios-padrão, a matriz proporção foi convertida em uma matriz-Z. Para a correção dos escores-Z foi adicionado o fator de correção $+2,5$ a fim de eliminar os valores negativos. Para o cálculo dos coeficientes de ponderação, aplicou-se o fator de correção ao valor da média dos escores-Z. Essa parte identifica a ordem de importância atribuída pelos enfermeiros do período noturno a cada componente do trabalho do ISP no que se refere à satisfação profissional.

Na análise da Parte B, primeiramente os itens foram agrupados por componente. Posteriormente, foram criadas matrizes de distribuição de freqüências das respostas por componente e, nesse momento, foi realizada a inversão dos escores dos itens positivamente ordenados. Isso significa que o escore 7 foi atribuído para concorda inteiramente. Em cada matriz foi realizada a soma dos valores numéricos 
de cada resposta, para cada componente, que foi dividido pelo número de questões de cada componente, resultando em um escore médio. Essa parte avalia a satisfação profissional percebida por esses enfermeiros em relação ao seu trabalho atual.

O nível real de satisfação profissional foi obtido a partir da medida ponderada entre a Parte A e a Parte B, ou seja, foi realizada a multiplicação do coeficiente de ponderação pelo escore médio de cada componente, o que resultou no escore ajustado por componente.

Para preservar a identidade dos participantes desta pesquisa, os questionários foram identificados, pela pesquisadora, pela letra $\mathrm{E}$ (Enfermeiro), seguidos do número de ordem da entrega (E1, E2, E3,...).

\section{RESULTADOS E DISCUSSÃO}

Como evidenciam os dados apresentados na Tabela 1, os enfermeiros do período noturno da instituição pesquisada são, majoritariamente, do sexo feminino (90,48\%), com faixa etária maior que 41 anos de idade $(64,28 \%)$, estado civil casado ou com companheiro $(64,29 \%)$ e com filhos $(80,96 \%)$. Dados semelhantes foram encontrados em outros estudos realizados com trabalhadores de enfermagem que atuavam no período noturno. ${ }^{8-9}$

Tabela 1 - Dados sociodemográficos dos enfermeiros $(n=42)$ que atuam no período noturno de um Hospital Universitário. RS, 2008

\begin{tabular}{lrr}
\hline Dados sóciodemográficos & $\mathbf{n}$ & \multicolumn{1}{c}{$\%$} \\
\hline Sexo & 4 & 9,52 \\
$\quad$ Masculino & 38 & 90,48 \\
$\quad$ Feminino & & \\
Faixa etária & 3 & 7,14 \\
20 a 30 & 12 & 28,57 \\
31 a 40 & 27 & 64,28 \\
$>41$ & & \\
Estado civil & 8 & 19,05 \\
$\quad$ Solteiro & 27 & 64,29 \\
$\quad$ Casado/companheiro & 7 & 16,67 \\
$\quad$ Separado/divorciado & & \\
Filhos & 8 & 19,05 \\
$\quad$ Não tem filhos & 12 & 28,57 \\
1 filho & 16 & 38,10 \\
2 filhos & 6 & 14,29 \\
3 filhos & \\
Opção por trabalhar no período noturno & \\
$\quad$ Sim & 38 & 90,48 \\
$\quad$ Não & 4 & 9,52 \\
Tempo de serviço noturno na instituição & & \\
1 a 5 & 13 & 30,95 \\
6 a 10 & 9 & 21,43 \\
11 a 15 & 8 & 19,05 \\
$>15$ & 12 & 28,57 \\
\hline
\end{tabular}

A percentagem expressiva de trabalhadores do sexo feminino dentre os enfermeiros é dado recorrente nos estudos nacionais., ${ }^{7,10-11}$ A questão de gênero na Enfermagem pode ser preponderante no enfrentamento dos conflitos no trabalho. ${ }^{1}$

Devido à faixa etária predominante, maior do que 41 anos, pode-se pressupor que sejam trabalhadores com relativa experiência de vida e maturidade. A relativa experiência de vida pode ser um fator que contribui para a maior tranqüilidade na tomada de decisões no trabalho e na vida pessoal. Outro dado aponta para o elevado número de enfermeiros com mais de 41 anos, o que é relevante, uma vez que, com o passar do tempo, o exercício do trabalho no noturno pode desencadear nesse trabalhador a mudança no cronotipo.

Cronotipo é uma propriedade do sistema de temporização circadiana. Desse modo, o fato de dormir tarde e acordar mais tarde é preferido pelo cronotipo vespertino; dormir cedo e acordar cedo é preferido pelos matutinos. ${ }^{13}$

A mudança no cronotipo poderá levar ao desenvolvimento de complicações à saúde e ao envelhecimento precoce do trabalhador. Pessoas que atuam no período noturno podem ter a idade como fator de risco adicional para o desenvolvimento de problemas de saúde e do denominado envelhecimento funcional precoce. ${ }^{12}$

Além disso, os dados da Tabela 1 revelam outros dois fatores que podem repercutir na qualidade de vida dos enfermeiros desse estudo: o fato de estar casado ou conviver com o companheiro associado à presença de filhos. Esses fatores podem desfavorecer o descanso após o plantão noturno, seja pelo cuidado dispensado, seja pelos ruídos.

Identifica-se na Tabela 1 que a maioria dos enfermeiros $(90,48 \%)$ optou por esse turno de trabalho. Ao serem questionados sobre as justificativas da opção, os enfermeiros informaram: a maior disponibilidade para a família, a menor freqüência de deslocamento para o hospital, a possibilidade de ter outro emprego, a experiência prévia como acadêmico de enfermagem ou como profissional no serviço noturno, o menor fluxo de pessoas, o ambiente mais calmo no noturno e o fato de poder realizar as atividades do lar. Dados semelhantes foram encontrados em outros estudos sobre o serviço noturno da enfermagem. ${ }^{8,14-15}$

O fato de poder escolher seu turno de trabalho pressupõe que o indivíduo tenha razões e necessidades que amparem essa escolha, o que poderá favorecer sua satisfação profissional. Desse 
modo, um trabalho livremente escolhido oferece, na maioria das vezes, vias de descarga mais adaptadas às necessidades, e o trabalho torna-se um meio de relaxamento a tal ponto que, uma vez a tarefa terminada, o trabalhador se sente melhor do que antes de tê-la começado. ${ }^{3}$

Quanto ao tempo de serviço noturno na instituição pesquisada, é possível observar dois grupos de trabalhadores: o primeiro grupo compreende os trabalhadores que tinham entre um e cinco anos de serviço noturno na instituição e que estavam experimentando uma adaptação tanto na vida social quanto no corpo, na mente e no ritmo biológico. O segundo grupo eram aqueles com mais de 15 anos de serviço noturno na instituição e que, possivelmente, já experimentavam alterações no ritmo biológico, com elevação das possibilidades de cronificação dos sinais e sintomas (Tabela 1).

Nesse sentido, há referência de que o tempo de serviço é proporcional à exposição dos trabalhadores aos fatores de risco físico, biológico, ergonômico, psicossocial e químico, aspectos essenciais na saúde do trabalhador e na sua satisfação com o trabalho. ${ }^{16}$

Em relação ao questionário do ISP, ressaltase que os dados obtidos a partir do escore da Escala de Atitudes (Parte B) foram submetidos à análise da consistência interna por meio da determinação do alfa de Cronbach. Partindo-se da premissa do alfa de Cronbach de que todas as correlações entre as variáveis sejam positivas e, portanto, as variáveis que apresentarem correlação negativa devem ser excluídas por violarem o modelo ${ }^{17}$ dois itens do componente normas organizacionais foram excluídos da análise. Após a exclusão, foi recalculado o alfa de Cronbach que, para este estudo, o valor do alfa geral da escala foi de 0,86 , sendo o mesmo considerado satisfatório.

A partir dos dados obtidos por meio da Parte A, foi criada uma matriz de freqüência absoluta que relacionou quantas vezes cada componente foi escolhido em relação aos outros componentes, conforme apresenta a Tabela 2.

Tabela 2 - Matriz de freqüências absolutas das respostas da Parte A $(n=42)$. RS, 2008

\begin{tabular}{|c|c|c|c|c|c|c|}
\hline & \multicolumn{6}{|c|}{ Mais importante } \\
\hline & SP & NO & $\mathbf{R}$ & RT & I & A \\
\hline$\stackrel{ \pm}{ \pm}$ Status Profissional (SP) & - & 21 & 29 & 31 & 31 & 38 \\
\hline స్ Normas Organizacionais (NO) & 21 & - & 24 & 29 & 34 & 28 \\
\hline 言 Remuneração (R) & 13 & 18 & - & 24 & 26 & 30 \\
\hline . & 11 & 13 & 18 & - & 22 & 34 \\
\hline Interação (I) & 11 & 8 & 16 & 20 & - & 23 \\
\hline$\sum^{\infty}$ Autonomia $(A)$ & 4 & 14 & 12 & 8 & 19 & - \\
\hline
\end{tabular}

No que se refere à satisfação profissional dos enfermeiros da pesquisa, o componente mais escolhido foi autonomia (38) e o menos escolhido foi status profissional (4).

Na seqüência, a matriz de freqüência absoluta foi convertida em matriz de proporção por meio da divisão do número de vezes que o componente foi escolhido pela população do estudo $(n=42)$, (Tabela 3).

Tabela 3 - Matriz de proporção das respostas da Parte A (n = 42). RS, 2008

\begin{tabular}{|c|c|c|c|c|c|c|c|}
\hline & & \multicolumn{6}{|c|}{ Mais importante } \\
\hline & & SP & NO & $\mathbf{R}$ & RT & $I$ & A \\
\hline \multirow{6}{*}{ 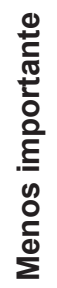 } & Status Profissional (SP) & - & 0,5000 & 0,6905 & 0,7381 & 0,7381 & 0,9048 \\
\hline & Normas Organizacionais (NO) & 0,5000 & - & 0,5714 & 0,6905 & 0,8095 & 0,6667 \\
\hline & Remuneração (R) & 0,3095 & 0,4286 & - & 0,5714 & 0,6190 & 0,7143 \\
\hline & Requisitos do Trabalho (RT) & 0,2619 & 0,3095 & 0,4286 & - & 0,5238 & 0,8095 \\
\hline & Interação (I) & 0,2619 & 0,1905 & 0,3810 & 0,4762 & - & 0,5476 \\
\hline & Autonomia $(A)$ & 0,0952 & 0,3333 & 0,2857 & 0,1905 & 0,4524 & - \\
\hline
\end{tabular}

Para a conversão das proporções em desviospadrão, a matriz proporção foi convertida em uma matriz dos escores-Z. Para o cálculo dos coeficientes de ponderação, aplicou-se o fator de correção de $+2,5$ ao valor da média dos escores$Z$, para eliminar os valores negativos, conforme mostra a Tabela 4. 
Tabela 4 - Matriz dos escores-Z das respostas obtidas por meio da Parte A do Índice de Satisfação Profissional, $(n=42)$, mostrando o coeficiente de ponderação. RS, 2008

\begin{tabular}{|c|c|c|c|c|c|c|c|}
\hline & & \multicolumn{6}{|c|}{ Mais importante } \\
\hline & & SP & NO & $\mathbf{R}$ & RT & I & A \\
\hline \multirow{6}{*}{ 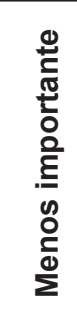 } & Status Profissional (SP) & - & 0,0000 & 0,9050 & 1,1313 & 1,1313 & 1,9232 \\
\hline & Normas Organizacionais (NO) & 0,0000 & - & 0,3394 & 0,9050 & 1,4707 & 0,7919 \\
\hline & Remuneração (R) & $-0,9050$ & $-0,3394$ & - & 0,3394 & 0,5656 & 1,0181 \\
\hline & Requisitos do Trabalho (RT) & $-1,1313$ & $-0,9050$ & $-0,3394$ & - & 0,1131 & 1,4707 \\
\hline & Interação (I) & $-1,1313$ & $-1,4707$ & $-0,5656$ & $-0,1131$ & - & 0,2263 \\
\hline & Autonomia $(A)$ & $-1,9232$ & $-0,7919$ & $-1,0181$ & $-1,4707$ & $-0,2263$ & - \\
\hline \multicolumn{2}{|c|}{ Soma } & $-5,0907$ & $-3,5070$ & $-0,6788$ & 0,7919 & 3,0544 & 5,4301 \\
\hline \multicolumn{2}{|c|}{ Média } & $-1,0181$ & $-0,7014$ & $-0,1358$ & 0,1584 & 0,6109 & 1,0860 \\
\hline \multicolumn{2}{|c|}{ Fator de correção } & 2,5 & 2,5 & 2,5 & 2,5 & 2,5 & 2,5 \\
\hline \multicolumn{2}{|c|}{ Coeficiente de ponderação } & 1,4819 & 1,7986 & 2,3642 & 2,6584 & 3,1109 & 3,5860 \\
\hline
\end{tabular}

O coeficiente de ponderação, em destaque na Tabela 4, indica a ordem de importância atribuída pelos enfermeiros a cada um dos componentes do ISP. Desse modo, percebe-se que o componente considerado mais importante no que se refere à satisfação profissional para os enfermeiros desta pesquisa é autonomia.

A Tabela 5 apresenta, com ênfase, o escore ajustado por componente, que foi obtido por meio da multiplicação do coeficiente de ponderação pelo escore médio de cada componente. Assim, foi possível identificar que os enfermeiros que atuavam no período noturno tinham maior nível real de satisfação profissional com a autonomia que possuíam para a realização das suas atividades. Esse dado é semelhante aos encontrados por outros autores., ${ }^{7,18}$

O componente normas organizacionais foi identificado como sendo o de menor nível real de satisfação profissional, dado que converge com os resultados de outros estudos brasileiros. ${ }^{7,11,18}$

Tabela 5 - Estatística descritiva dos componentes do Índice de Satisfação Profissional para os enfermeiros do período noturno. RS, 2008

\begin{tabular}{lccc}
\hline Componente & $\begin{array}{c}\text { Coeficiente } \\
\text { de } \\
\text { ponderação }\end{array}$ & $\begin{array}{c}\text { Escore } \\
\text { médio }\end{array}$ & $\begin{array}{c}\text { Escore } \\
\text { ajustado }\end{array}$ \\
\hline Autonomia & 3,59 & 4,67 & 16,73 \\
Interação & 3,11 & 4,44 & 13,81 \\
Requisitos do trabalho & 2,66 & 3,56 & 9,46 \\
Status profissional & 1,48 & 5,57 & 8,24 \\
Remuneração & 2,36 & 2,59 & 6,11 \\
Normas organizacionais & 1,80 & 3,20 & 5,76 \\
\hline
\end{tabular}

A autonomia foi o componente em que os enfermeiros estavam realmente mais satisfeitos profissionalmente. Esse era um dado esperado, uma vez que, no serviço noturno, há menor fluxo de profissionais e redução dos serviços de apoio, o que possibilita e também pressiona o enfermeiro a participar efetivamente das decisões.

Supõe-se que o enfermeiro que tem oportunidades de exteriorizar suas opiniões, atuando de forma efetiva no processo de tomada de decisão, tem retorno do seu trabalho, o que pode contribuir para a sua satisfação pessoal e profissional. Desse modo, propiciar a autonomia aos trabalhadores por meio do processo de pensar-fazer seu trabalho e ampliar o grau de abertura aos processos de criação, permite alcançar o prazer no trabalho. ${ }^{19}$ Assim, "quando o sofrimento pode ser transformado em criatividade, ele faz uma contribuição que beneficia a identidade" 3:137

Desse modo, a constante busca pela autonomia profissional repercute na construção da identidade profissional do enfermeiro, que, por meio do conhecimento científico, pode enfrentar situações que requeiram avaliação e tomada de decisão. ${ }^{4}$

A autonomia pressupõe a busca de informações, conhecimentos técnico-científicos, criatividade e controle sobre o processo de trabalho. A enfermagem autônoma "utiliza uma conduta que lhe permite estabelecer, conscientemente, os limites e alcances máximos do seu fazer diário porque, supostamente, conhece os espaços em que tem direito de atuar". ${ }^{20: 92}$

A interação, como segundo componente de maior nível real de satisfação profissional, também foi um dado esperado, uma vez que o trabalho 
Silva RM, Beck CLC, Guido LA, Lopes LFD, Santos JLG

interativo e em equipe no período noturno é favorecido pelas especificidades desse turno. $\mathrm{O}$ desafio de manter-se acordado associado às 12 horas de convívio com os colegas, oportunizam a socialização de angústias e inquietações, assim como de alegrias, saberes e conquistas, o que pode repercutir favoravelmente no ambiente de trabalho e na satisfação profissional.

Desse modo, devido às características do trabalho hospitalar, as relações interpessoais assumem papel importante na concepção do trabalho e no sentido que poderá ser conferido a ele, uma vez que esse congrega diversos trabalhadores com visões de mundo e do trabalho assistencial diversificado. ${ }^{21}$

Com relação às normas organizacionais, há referência de que o enfermeiro, além de participar efetivamente das etapas da organização do trabalho, tem a responsabilidade de manter e reproduzir as normas organizacionais da instituição, o que pode, sob algumas circunstâncias, levá-lo a trair seus princípios e valores, gerando insatisfação e sofrimento. ${ }^{7}$ É o que pode ter contribuído para que este fosse o componente de menor nível real de satisfação profissional.

Os componentes requisitos do trabalho, status profissional e remuneração, ocuparam, respectivamente, o terceiro, quarto e quinto lugar na identificação da real satisfação do grupo pesquisado, o que significa dizer que são componentes que, para este estudo, exercem menor influência sobre a satisfação profissional dos enfermeiros.

A satisfação profissional é um fenômeno subjetivo e pode ser bastante específico para determinado ambiente de trabalho. Em vista disso, essa é uma das razões para implementar estudos de satisfação, seja para criar ou avaliar mudanças dentro de uma organização. ${ }^{22}$

Desse modo, os dados apontam que a real satisfação profissional dos enfermeiros estava diretamente atrelada à possibilidade de tomada de decisão e de liberdade de escolhas, e inversamente relacionava-se com as normas estabelecidas pela instituição.

\section{CONCLUSÕES}

A partir da análise dos dados e considerando o objetivo proposto para este estudo, os resultados encontrados permitiram as seguintes conclusões:

- os enfermeiros do período noturno da instituição pesquisada são a maioria do sexo feminino $(90,48 \%)$, com faixa etária maior que 41 anos de idade (64,28\%), estado civil casado ou com companheiro $(64,29 \%)$, com filhos $(80,96 \%)$ e que escolheram trabalhar nesse turno $(90,48 \%)$;

- quanto ao nível real de satisfação profissional dos enfermeiros do grupo estudado do período noturno em relação aos componentes do ISP, verificou-se que esses trabalhadores estavam mais satisfeitos com o componente autonomia seguido pelo componente interação, requisitos do trabalho, status profissional e remuneração;

- o componente com o qual os enfermeiros do grupo estudado têm menor nível real de satisfação profissional foi as normas organizacionais.

Diante disso, constata-se que a satisfação profissionale influenciada por uma série de fatores e está diretamente relacionada à subjetividade do trabalhador. Neste estudo, o fato dos componentes autonomia e normas organizacionais terem se destacado, respectivamente, como sendo o que proporciona maior e menor satisfação profissional, permite uma reflexão interessante quanto aos aspectos que vem sendo priorizados pela gestão das instituições hospitalares, ou seja, a dedicação à elaboração de normas e rotinas no intuito de organizar e padronizar o processo de trabalho.

Entretanto, é importante salientar que normas e rotinas são implementadas, geralmente, por profissionais diferentes dos que as elaboraram e em um contexto dinâmico que nem sempre corresponde a parâmetros pré-estabelecidos, fator esse que pode influenciar a satisfação no trabalho.

Ressalta-se que o fato de ter sido realizado em um Hospital Universitário pode ser uma limitação do presente estudo. Assim, sugere-se a realização de novos estudos, em especial em instituições privadas, sobre a saúde do trabalhador de enfermagem que atua no noturno e sua relação com o trabalho, a fim de possibilitar possíveis comparações com os resultados deste estudo.

Por fim, considerando a importância do trabalho para o ser humano e a carência de estudos que envolvam a temática, evidencia-se que esta é uma área de investigação que precisa ser desenvolvida, uma vez que, a compreensão das questões relacionadas à satisfação profissional do enfermeiro que exerce suas atividades no período noturno pode sugerir ações interventivas com vistas à melhoria da sua qualidade de vida, da assistência prestada e da satisfação profissional.

\section{REFERENCIAS}

1. Beck CLC. O sofrimento do trabalhador: da banalização a re-significação ética na organização da 
enfermagem [tese]. Florianópolis (SC): Universidade Federal de Santa Catarina. Programa de PósGraduação em Enfermagem; 2001.

2. Marqueze EC, Moreno CR de C. Satisfação no trabalho: uma breve revisão. Rev Bras Saúde Ocupacional. 2005; 30(112):69-79.

3. Dejours C. A carga psíquica do trabalho. In: Dejours C, Abdoucheli E, Jaye C, organizadores. Psicodinâmica do trabalho: contribuições da Escola Dejouriana à análise da relação prazer, sofrimento e trabalho. São Paulo (SP): Atlas; 2007. p.21-32.

4. Silva RM. Satisfação profissional dos enfermeiros de um hospital universitário no trabalho noturno [dissertação]. Santa Maria (RS): Universidade Federal de Santa Maria. Programa de Pós Graduação em Enfermagem; 2008.

5. Minayo MCS, Assis SG, Souza ER, organizadores. Avaliação por triangulação de métodos. Rio de Janeiro (RJ): Fiocruz; 2005.

6. Ministério da Saúde (BR). Resolução No 196 de 10 de outubro de 1996: diretrizes e normas regulamentadoras de pesquisa em seres humanos. Brasília (DF): MS; 1996.

7. Lino MM. Satisfação profissional entre enfermeiras de UTI: adaptação transcultural do Index Work Satisfaction (IWS) [dissertação]. São Paulo (SP): Universidade de São Paulo. Programa de PósGraduação em Enfermagem; 1999.

8. Moraes DR. Saúde e trabalho em turno noturno: possibilidades e limites na avaliação de auxiliares de enfermagem - um estudo de caso [dissertação]. Porto Alegre (RS): Universidade Federal do Rio Grande do Sul. Programa de Pós-Graduação em Psicologia Social e Institucional; 2004.

9. Magalhães AMM, Martins CMS, Falk MLR, Fontes $\mathrm{CV}$, Nunes VB. Perfil dos profissionais enfermeiros no turno noturno do Hospital de Clínicas de Porto Alegre. Rev HCPA 2007; 27(2):16-20.

10. Guido LA. Stress e coping entre enfermeiros de centro cirúrgico e recuperação anestésica [tese]. São Paulo (SP): Universidade de São Paulo. Programa de Pós-Graduação em Enfermagem; 2003.

11. Lino MM. Qualidade de vida e satisfação profissional de enfermeiras de unidade de terapia intensiva [tese]. São Paulo (SP): Universidade de São Paulo. Programa de Pós-Graduação em Enfermagem; 2004.
12. Moreno CRC, Fischer FM, Rotenberg L. A saúde do trabalhador na sociedade 24 horas. São Paulo Perspec. 2003 Jan-Mar; 17(1):34-46.

13. Alam MF, Tomasi E, Lima MS, Areas R, MennaBarreto L. Caracterização e distribuição de cronotipos no sul do Brasil: diferenças de gênero e estação de nascimento. J Bras psiquiatr. 2008; 57(2):83-90.

14. Garcia MJ. O trabalho noturno e suas implicações para a equipe de enfermagem: estudo em unidade de clínica cirúrgica [dissertação]. Rio de Janeiro (RJ): Universidade Estadual do Rio de Janeiro. Programa de Pós-Graduação em Enfermagem; 2004.

15. Oliveira MM. Alterações psicofisiológicas dos trabalhadores de Enfermagem no serviço noturno [dissertação]. Rio de Janeiro (RJ): Universidade Estadual do Rio de Janeiro. Programa de PósGraduação em Enfermagem; 2005.

16. Schmidt DRC. Qualidade de vida e qualidade de vida no trabalho de profissionais de enfermagem atuantes em unidades de bloco cirúrgico [dissertação]. São Paulo (SP): Universidade de São Paulo. Programa de Pós-Graduação em Enfermagem; 2004.

17. Pereira JCR. Análise de dados qualitativos: estratégias metodológicas para as Ciências da Saúde, Humanas e Sociais. $3^{\text {a }}$ ed. São Paulo (SP): Edusp; 2001.

18. Campos RM. Satisfação da equipe de enfermagem do serviço de atendimento móvel às urgências (SAMU) no ambiente de trabalho [dissertação]. Natal (RN): Universidade Federal do Rio Grande do Norte. Programa de Pós-Graduação em Enfermagem; 2005.

19. Barros RB, Barros MEB. Da dor ao prazer no trabalho. In: Santos-Filho SB, Barros MEB, organizadores. Trabalhador da saúde: muito prazer! Protagonismo dos trabalhadores na gestão do trabalho em saúde. Ijuí (RS): Unijuí; 2007. p.61-71.

20. Souza JG. Autonomia e cidadania na enfermagem. Texto Contexto Enferm. 2000 Ago-Dez; 9(3):86-99.

21. Souza NVDO, Lisboa MTL. Ritmo de trabalho: fator de desgaste psíquico da enfermeira. Esc Anna Nery Rev Enferm. 2005 Ago; 9(2):229-36.

22. Stamps PL. Using the index of work satisfaction in an organizational setting. In: Stamps PL, organizador. Nurses and work satisfaction: an index for measurement. $2^{\mathrm{a}}$ ed. Chicago (US): Health Administration Press; 1997. p.243-55. 